\title{
Ein unruhiges Jahr geht zu Ende
}

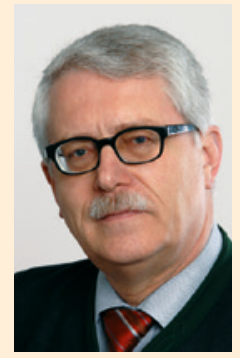

Liebe Leserinnen und Leser der FTR,

mit dem vorliegenden Heft halten Sie die letzte Ausgabe der FTR für dieses Jahr in Ihren Händen. In der Rückschau stelle ich für mich fest, dass ich mich kaum an ein unruhigeres Jahr erinnern kann: Es gibt wenige ermutigende Zeichen des Friedens auf der Welt. Innerhalb unseres Landes steht der gesellschaftliche Zusammenhalt unter erheblicher Spannung. Für die 6 Fachgesellschaften, welche die FTR herausgeben, bedeutet der Zustand der Welt eine zusätzliche Herausforderung - sind doch unsere Mitglieder aus ihrem Selbstverständnis an anderen Menschen, Kulturen und Landschaften besonders interessiert. Das gilt auch für die meisten der Ratsuchenden in unseren Praxen. Ich hoffe auf ein ruhigeres und friedlicheres 2016.

Die Kasuistik in dieser Ausgabe beschreibt einen Fall, der sich auch für ein hochangesehenes Institut wie das Hamburger Bernhard-Nocht-Institut als diagnostische Herausforderung darstellte. Ein 29-jähriger Rucksacktourist war nach 4 Wochen Thailand mit Fieber, Schüttelfrost und Gliederschmerzen in der tropenmedizinischen Ambulanz vorstellig geworden. Wegen der unspezifischen Symptomatik konnte der Fall allein klinisch nicht aufgelöst werden. Christoph Scheurich, Dennis Tappe, Nina Raabe und Camilla Rothe zeigen auf, wie sie schließlich zur Diagnose einer Doppelinfektion mit Leptospiren und Dengueviren kamen - lehrreich!

Eine Übersicht, auf die viele von uns schon lange gewartet haben, bietet Jörg Siedenburg in seinem Artikel zum Thema „Ärzte und Fliegen“. Dabei geht es sowohl um die ärztliche Betreuung und Medikamenteneinnahme der Piloten als auch des Kabinenpersonals. Neben der Darstellung der gesetzlichen Vorgaben, Hinweisen auf Indikationen, Karenzzeiten und Beschränkungen ist die Arbeit eine wichtige und beklemmend aktuelle Hilfestellung für Fliegerärzte und behandelnde Ärzte.

„Wir sind Geschöpfe der Gravitation“ - mit diesem Zitat von Kirsch und Grunga beginnt Carla Ledderhos ihre Übersicht der historischen und aktuellen Forschung mit künstlicher Schwerkraft. Sie lässt keinen Zweifel, dass die Ergebnisse einen wichtigen Beitrag zur Flugsicherheit leisten. Ohne die experimentellen Möglichkeiten, in modernen Humanzentrifugen permanente künstliche Schwerkraft zu erzeugen, wird der geplante Flug zum Mars nicht möglich sein. Nicht nur wegen dieses Einblicks in die aktuelle Forschung, sondern auch wegen der historischen Betrachtung zu den Belastungen der Piloten in den Weltkriegen, empfehle ich die Lektüre auch für die Nichtexperten.

Gerne lenke ich im Panorama Ihr Augenmerk zunächst auf den Beitrag zur Diagnostik des Q-Fiebers. Autoren sind Dimitrios Frangoulidis und meine ehemalige Mitarbeiterin Silke Fischer. Meine eigenen Erfahrungen zeigen, dass man gelegentlich einige Irrwege hinter sich lassen muss, bis die Diagnose Q-Fieber steht beziehungsweise daran gedacht wird.

Aufschlussreich sind die Ausführungen von Michael Karg, Christoph Jänig und Christian Ottomann zur Ausbildung und den vielfältigen Einsatzmöglichkeiten als Schiffsarzt bei der deutschen Marine. Neben einer fundierten klinischen Weiterbildung, vorzugsweise in Innere Medizin und Chirurgie, ergänzt durch gründliche Kenntnisse in der Notfallmedizin, werden Einfühlungsvermögen, Organisationstalent und Teamfähigkeit benötigt. Dann eröffnet sich ein reiches Betätigungsfeld. Fühlen Sie sich angesprochen?

Mit den besten Wünschen für die kommenden Festtage und das Neue Jahr

Prof. Dr. Günter Schmolz 